\title{
Effective Temperatures and Gravities for O-type Stars Determined from High Precision Line Profiles and Wind-Blanketed Model Atmospheres
}

\author{
B. Bohannan, S.A. Voels, D.C. Abbott, and D.G. Hummer \\ Joint Institute for Laboratory Astrophysics \\ Astrophysical, Planetary and Atmospheric Sciences \\ University of Colorado, Boulder, Colorado, U.S.A.
}

\section{Introduction}

Analysis of line profiles obtained with astronomical instrumentation capable of high signal-to-noise ratio spectroscopy have contributed significant new precision to the determination of the basic stellar parameters of hot, luminous, mass-loosing stars. Accurate measurement of such stellar properties as effective temperature and helium abundance for stars of spectral type $\mathrm{O}$ and early $\mathrm{B}$ is important not only to the physics of these stars but also to the environment in which they are located. The overall goals of the work we summarize here are to refine the spectral-type $v s$. temperature calibration for the most massive stars and to determine helium abundances for stars that are loosing mass at a rate sufficiently high to affect their evolution. Details of our procedures are described in the analysis of $\zeta$ Puppis by Bohannan et al. (1986).

For stars hotter than roughly $35,000 \mathrm{~K}$, accurate values of effective temperature cannot be determined from the observed continuum flux distribution (Hummer et al. 1988); instead, effective temperature, surface gravity and helium abundance must be simultaneously measured through an analysis of high-precision line profiles using model atmospheres that contain the best available physics. The theoretical atmosphere used in the analysis we describe here assumes non-local thermodynamic equilibrium and includes the effect of heating of the photosphere by radiation backscattered by the stellar wind, the wind-blanketing effect (Hummer 1982). A description of the wind-blanketed model atmospheres is given by Abbott and Hummer (1985).

To achieve results of sufficient accuracy that will contribute significantly to such areas as the study of the initial mass function and the observational resolution of various theories of stellar structure and evolution, our analysis requires observations of high photometric precision. For stars with effective temperature on the order of $40,000 \mathrm{~K}$, the wings of $\mathrm{H} \gamma$ must be determined with an accuracy of $0.2 \%$ to obtain gravities with an error of $0.1 \mathrm{dex}$; to measure temperatures with an error of $5 \%$ requires that the core-wing region of neutral and ionized helium lines must be observed at the $1 \%$ level.

\section{The Observations}

Photometric accuracy at the levels dictated by our goals are not possible with photographic techniques, but can be obtained with currently available electronic detectors capable of linear response over a wide dynamic range. For a number of $\mathrm{O}$ and early B-type stars with mass loss rates well-determined from radio measures, we have 
observed essentially all of the hydrogen and helium lines in the wavelength region $3900 \AA$ to $7100 \AA$ with the Kitt Peak National Observatory coudé-feed telescope and coudé spectrograph equipped for most of our runs with a TI 3-phase 800x800-pixel CCD. The spectral resolution is $0.30 \AA$ in the blue and $0.45 \AA$ in the red.

The photometric precision of our line profiles is $0.5 \%$ or better. We have found that, because of calibration of flat-fielding effects, the photometric precision of the observed line profiles is less than that predicted from photon statistics. Apparently the CCD in the coude spectrograph is not identically illuminated by the starlight as by the flat-field lamp used to remove pixel-to-pixel variations. Our present technique is to trail the star along the slit; in this way we spread the spectrum over 10 to 40 columns of the $\mathrm{CCD}$ in an attempt to average residual fringing effects. We achieve with less observing time a higher signal-to-noise ratio spectrum with the trailing strategy than was possible by adding together spectra taken at different grating settings. However, there is still some residual fringing remaining which we attribute to fringing effects that are strongly wavelength dependent and which lie perpendicular to the dispersion, effects that do not average out when the spectrum is trailed. Additionally, there is some uncertainty in the profiles associated with fitting the continuum to the very broad absorption line profiles found in early-type stars.

\section{The Model Atmospheres}

The wind-blanketed model atmospheres used in our analysis are those of Mihalas (1972) with a modified upper boundary which allows for back-scattering of radiation by an outflowing stellar wind. The photospheric model is based upon assumptions of radiative and hydrostatic equilibrium, plane-parallel geometry, non-local thermodynamic statistical equilibrium and contains only hydrogen and helium. The stellar wind model includes radiation from the photospheric model and multiscattering by overlapping lines and by electrons. Some 10,000 lines between $200 \AA$ and $10,000 \AA$ are included. We use the mass loss rate set by the radio flux and the terminal velocity as measured from ultraviolet resonance lines.

In general, we find a good fit by the model line profiles to the observations (for examples, see Bohannan et al. 1986 and 1988). However, we do not model well those lines formed high in the atmosphere where the atmosphere may be spherically extended and possibly not static.

\section{The Black-Art of Line Profile Fitting}

To achieve the most accurate results possible, one cannot use equivalent widths of just a few lines to determine basic stellar parameters. Rather, effective temperature, gravity and helium abundance must be set simultaneously to as many lines as feasible. In our work, we include a large number of transitions to overcome the effects of unresolved blends, of observational errors and of modeling deficiencies. For a starting point in temperature, we use HeI $\lambda 4471 \AA$ and HeII $\lambda 4542 \AA$ profiles; for a starting gravity, $\mathrm{H} \gamma \lambda 4340 \AA$. We then vary the temperature and gravity until we get a good fit to all of the hydrogen and helium transitions observed, usually about 10 lines in all.

Through a plot of depth of formation, we identify those transitions formed high in the atmosphere which may not produce good fits. In general, we find that strong lines of the dominate ionization stage of helium are not well modeled by our code. For example, lines like HeII $\lambda 5411 \AA$ and HeII $\lambda 4542 \AA$ in $\zeta$ Puppis (O4If) and HeI $\lambda 5876 \AA$ and HeI $\lambda 5411 \AA$ in $\alpha$ Cam (O9.5Ia), transitions formed high in the respective atmospheres, are not fit with the same precision as other transitions of helium. Note that this discrepancy 
affects the two lines, HeII $\lambda 4542 \AA$ and $\mathrm{HeI} \lambda 4471 \AA$, that are the fundamental temperature criterion in defining the spectral classification of 0 -type stars. Since we are able to fit lines formed deep near the continuum, we do not feel that this deficiency seriously affects the accuracy of the stellar parameters we measure.

To determine the helium fraction, we use a self-consistency test on the effective temperature and helium fraction using equivalent widths of all of the neutral helium and unblended ionized helium lines. We iterate with a set of temperature, gravity and helium abundance until we get a consistent fit to all of the lines formed deep in the atmosphere.

\section{Discussion}

The table below summarizes our results to date. The temperature scale for evolved early O-type stars has been reduced some $20 \%$ over that of Conti (1973). For spectral type O4If, allowance for an appropriate gravity made possible by high precision line profiles has changed the temperature by $-8000 \mathrm{~K}$, inclusion in the model atmosphere of the effect of wind-blanketing accounted for a change of $-4500 \mathrm{~K}$, and the use of high precision line profiles and the analysis of many lines of helium produced a change of $+4000 \mathrm{~K}$. We estimate the errors of our stellar parameters as follows: in effective temperature, $\pm 1500 \mathrm{~K}$ at $\mathrm{O} 4$ and $\pm 1000 \mathrm{~K}$ at 09.5 ; in gravity, \pm 0.1 dex at 04 and \pm 0.05 dex at 09.5 ; in helium fraction, $[\mathrm{Y}], \pm 0.03$.

Adopted and Derived Stellar Parameters of Some Early-type Stars From High Precision Line Profiles and Wind-Blanketed Model Atmospheres

\begin{tabular}{|c|c|c|c|c|c|c|}
\hline Star & Sp. Type & $\log \mathrm{L} / \mathrm{L}_{\mathrm{O}}$ & $\log \mathbf{M} / \mathbf{M}_{\mathrm{O}}$ & $\mathrm{T}_{\mathrm{eff}}\left({ }^{\circ} \mathrm{K}\right)$ & $\log g$ & {$[\mathrm{Y}]$} \\
\hline$\zeta$ Pup & O4If & 6.00 & -5.3 & 42,000 & 3.5 & \\
\hline $9 \mathrm{Sgr}$ & O4V & 6.11 & -5.4 & 46,000 & 3.9 & \\
\hline$\alpha \mathrm{Cam}$ & O9.5Ia & 5.78 & -5.4 & 30,000 & 2.85 & \\
\hline$\zeta$ Ori & O9.5Ib & 5.77 & -5.8 & 31,000 & 3.2 & \\
\hline$\delta$ Ori & O9.5II & 5.60 & -6.3 & 33,000 & 3.4 & \\
\hline
\end{tabular}

The high helium abundance observed for $\zeta$ Puppis is consistent with the evolved nature of this star which has lost some $40 \mathrm{Mo}_{0}$ of material from its original mass of 70-90 Mo. A similar conclusion has been reached for $\alpha$ Cam. There is a hint from photographically derived equivalent widths of a large number of O-type stars that this result may hold true for many, if not all, evolved O-type stars.

\section{References:}

Abbott, D. C. and Hummer, D. G. 1985, Ap. J. 294, 286.

Bohannan, B., Abbott, D. C., Voels, S. A., Hummer, D. G. 1986, Ap. J. 308, 728.

Bohannan, B., Voels, S. A., Abbott, D. C., Hummer, D. G. 1988, submitted to Ap. J. Conti, P. S. 1973, Ap. J. 179, 161.

Hummer, D. G. 1982, Ap. J. 257, 724.

Hummer, D. G., Voels, S. A., Abbott, D. C., Bohannan, B. 1988, submitted to Ap.J.

Mihalas, D. 1972, NCAR TN/STR-76, Non-LTE Model Atmospheres of B and O Stars. 


\section{DISCUSSION}

EBBETS You mentioned that you did not see variability in aCam.

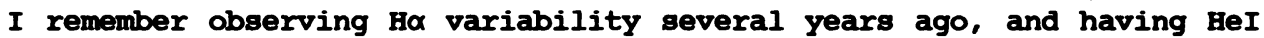
$\lambda 6678$ on the same spectra. This was an absorption line, and it did show night to night variations in profile.

BOHANNAN Ho and other "wind" lines are certainly variable in line strength. We have tried to concentrate on lines formed primarily in the photosphere. For example, we have multiple observations of $\mathrm{Hy}$ in ocam that appear to be constant in profile. We would like to believe that any bump and wiggles seen in line profiles have only a small effect on the temperatures and gravities that we derive.

EDVARDSSON Just how sensitive are your surface gravities to, say, a $2000 \mathrm{~K}$ error in the effective temperature of the star.

BOHANNAN We solve for temperature, gravity and helium abundance in an iterative fashion. The starting gravity comes from Hy the starting temperature from neutral and ionized helium lines. We then adjust the temperature and gravity until we get a best fit to all of the lines. The interaction between temperature and gravity depends strongly upon what spectral type star is under consideration. But there will not be a systematic error in gravity caused by an uncertainty in temperature because different lines have different sensitivities to temperature and to gravity. For aCam we have a sensitivity of $1000 \mathrm{~K}$ in effective temperature and 0.05 dex in gravity.

GRATTON What do you mean by $Y$ ?

BOHANNAN $[Y]$ here is the number fraction of helium.

GRIFPIN You said that co-adding spectra in order to increase $s / N$ "did not work". Could you please say why not ?

BOHANNAN Adding together CCD spectra taken at the same grating setting does not remove, rather strengthens, the effects of residual fringing. Adding together spectra at slightly different grating settings has the potential of achieving the highest $S / N$ but is inefficient of telescope time as it requires independent flat-field exposures at each grating setting. Our present technique is to trail the star over $10-40$ lines on the $\mathrm{CCD}$. However, some low level $(\simeq 0.28)$ residual fringing remains after extraction because fringing is a wavelength dependent phenomenon and any residual fringing present will tend to lie perpendicular to the dispersion. 